\title{
Effect of Various Plant Growth Regulators on Growth and Yield of Cotton (Gossypium hirsutum)
}

\author{
S.S. Sabale, G.R. Lahane* and S.J. Dhakulkar
}

Department of Genetics and Plant breeding, C. P. College of Agriculture, S. D. Agriculture University, Sardarkrushinagar-385506, Gujarat, India

*Corresponding author

\section{A B S T R A C T}

\begin{tabular}{|l|}
\hline K e y w o r d s \\
PGRs, Cotton, \\
Gossypium hirsutum \\
L., Yield.
\end{tabular}

\section{Introduction}

Cotton is a sub-tropical, perennial plant with indeterminate growth habit. Vegetative and reproductive growth occurs simultaneously where vegetative growth is necessary to support reproductive growth. The growth habits of these varieties/hybrids combined with high availability of nutrients, timely rainfall or irrigation and delayed fruit retention can encourage excessive vegetative growth. Excessive vegetative growth leads to severe production problems like fruit abortion, delayed maturity, boll rot and harvest difficulties. The physiological efficiency of a plant can be improved by prolonging photosynthesis, reducing photorespiration, better partitioning of photo assimilates, improving mineral ions uptake and stimulating nitrogen metabolism. All these processes are inter-linked through several interactions and influence growth and productivity.

Plant growth regulators have been found to influence these processes in one way or the other. Plant growth regulators are substances when added in small amounts modify the 
growth of plant usually by stimulating or inhibiting part of the natural growth regulation. They are considered as new generation of agrochemicals after fertilizers, pesticides and herbicides. Plant growth regulators are capable of increasing yield by 100-200 per cent under laboratory conditions, 10 - 15 per cent in the field conditions (Kiran Kumar, 2001).

Plant growth regulators like promoters, inhibitors or retardants play a key role in internal control mechanism of plant growth by interacting with key metabolic processes such as nucleic acid and protein synthesis. The most commonly used growth regulator in cotton is mepiquatchloride, which is an inhibitor of gibberellic acid. This curtails excessive vegetative growth and increases the yield.

Generally sowing of cotton in Gujarat is done at the end of May to first week of June, so there will be maximum number of bolls per plant at the end of August to first fortnight of September. From last few years weather pattern has changed and rainfall withdraw at the end of August. So cotton faces moisture stress at this period on contrast to this plant of cotton at that time requires maximum water and foods for the development of bolls. The drought at this time create internal hormones imbalance i.e. production of abscisic acid and ethylene inhibits the production of Auxins, Gibberellins and Cytokinins which results into abscission of leaves and squares and in severe condition also abscission of bolls and ultimately parawilt condition in cotton yield.

\section{Materials and Methods}

Geographically, Sardarkrushinagar campus of Sardarkrushinagar Dantiwada Agricultural University, where the experiment was laid out is situated at $24-19^{\prime}$ North latitude and $72^{\circ}-$ 10' East longitude with an altitude of 154.52 metre from the mean sea level. It represents the North Gujarat Agro-climatic Zone. The soil of the experimental field was loamy sand in texture, low in organic carbon (0.16) and available nitrogen (144), medium in available phosphorus (31) and high in available potash (283). Electrical conductivity was very low showing that the soil was free from salinity hazard (Table 1).

The experiments were carried out in FRBD (Factorial Randomized Block Design)design with three replications having the spacing 120 $\mathrm{x} 45 \mathrm{~cm}$. Treatment divided into two factor, 1) Factor A: Chemicals (C), 2) Factor B: Varieties (V): a) Bt. Cotton - Hybrid 6b) Non Bt. Cotton-G. Cot. Hybrid 12.

Hand-thinned to 5 to 6 plants per meter row when the seedlings had approximately three true leaves. The recommended dose of fertilizer to cotton is 160: $00: 00 \mathrm{~N}, \mathrm{P} 2 \mathrm{O} 5$ and $\mathrm{K} 2 \mathrm{O} \mathrm{kg} / \mathrm{ha}$. Among this $80 \mathrm{~kg} \mathrm{~N}$ was applied at the time of sowing as basal dose. A top dressing of $40 \mathrm{~kg} \mathrm{~N}$ each was applied at 30 DAS and 60 DAS.

Total eighteen treatment combinations were used. The details of treatments are as under

Three replications are utilized for recording observation for nondestructive analysis. Five plant in each plots were randomly selected from net rows, tagged and were used to determine Plant height $(\mathrm{cm})$, Days to flower initiation, Total no. of flowers opened per plant, Total no. of flowers abscission per plant and no. of bud abscission per plant. For destructive analysis plant sample were taken from three replications.

Five plants were randomly selected for this purpose in net plots and carefully uprooted with the help of shevel from a depth of $60 \mathrm{~cm}$ to determine Total dry weight of plant ( $\mathrm{g}$ plant-1), Leaf area per plant (dm2 plant-1), 
Leaf area index and Chlorophyll content (mg g-1 fresh weight). At the time of harvesting the tagged five plants utilized for observations recording and plants were harvested separately for recording Seed cotton yield (kg/ha), Biological yield (gm), Harvest index (\%), NAR (Net assimilative rate) $\left(\mathrm{g}^{-1} \mathrm{dm}^{-2}\right.$ day $\left.^{-1}\right)$ and RGR (Relative growth rate) $\left(\mathrm{g} \mathrm{g}^{-1}\right.$ day $\left.^{-1}\right)$. From each plot, plants were selected randomly, for recording physiological character Total dry weight of plant $\left(\mathrm{g}\right.$ plant $\left.^{-1}\right)$, Leaf area per plant $\left(\mathrm{dm}^{2}\right.$ plant $\left.^{-1}\right)$, Leaf area index, Chlorophyll content $\left(\mathrm{mg} \mathrm{g}^{-1}\right.$ fresh weight), Seed cotton yield ( $\mathrm{kg} / \mathrm{ha})$, Biological yield (gm), Harvest index (\%), NAR (Net assimilative rate) $\left(\mathrm{g}^{-1} \mathrm{dm}^{-2} \mathrm{day}^{-1}\right)$ and RGR (Relative growth rate) $\left(\mathrm{g} \mathrm{g}^{-1}\right.$ day $\left.^{-1}\right)$.

The data collected for all the characters were subjected to statistical analysis by adopting 'Analysis of Variance' techniques as described by Panse and Sukhatme (1978).

\section{Results and Discussion}

Interaction effect of different plant growth regulators on $\mathrm{Bt}$. cotton and local hybrid cotton plant height at 90 DAS

The effect of different plant growth regulators on Bt. cotton and local hybrid cotton at 90 DAS was found to be significant. Application of NAA $30 \mathrm{ppm}$ at 80 DAS to Bt. cotton recorded significantly higher plant height $(95.33 \mathrm{~cm})$. However, it was at par with $\mathrm{GA}_{3}$ $50 \mathrm{ppm}$ at 80 DAS $(93.00 \mathrm{~cm})$, NAA $30 \mathrm{ppm}$ at $60 \mathrm{DAS}(92.00 \mathrm{~cm})$ and $\mathrm{GA}_{3} 50 \mathrm{ppm}$ at 60 DAS $(89.67 \mathrm{~cm})$.

The lower plant height was recorded with MC $(84.33 \mathrm{~cm})$ while in local hybrid cotton NAA $30 \mathrm{ppm}$ at 60 DAS recorded significantly higher plant height $(92.08 \mathrm{~cm})$ compared to other treatments. However, it was at par with $\mathrm{GA}_{3} 50 \mathrm{ppm}$ at 60 DAS $(92.00 \mathrm{~cm})$, NAA 30 ppm at 80 DAS $(91.92 \mathrm{~cm}), \mathrm{GA}_{3} 50 \mathrm{ppm}$ at
80 DAS $(90.50 \mathrm{~cm})$, Urea $2 \%$ at 80 DAS $(88.00 \mathrm{~cm})$ and Urea $2 \%$ at 60 DAS $(84.00$ $\mathrm{cm}$ ) (Table 3.1).

Interaction effect of different plant growth regulators on Bt. cotton and local hybrid cotton for bud abscission at 90 DAS

The effect of different plant growth regulators on Bt. cotton and local hybrid cotton at 90 DAS was found to be significant. In Bt. cotton the number of bud abscission differed significantly among the treatments. Number of bud abscission was significantly less when application of NAA $30 \mathrm{ppm}$ at 80 DAS (4.83). However, it was at par with MC 200 ppm at 60 DAS (6.00), MC $200 \mathrm{ppm}$ at 80 DAS (6.50), Urea $2 \%$ at 60 DAS (6.17) and Urea $2 \%$ at 80 DAS (5.83). Significantly highest number of bud abscission was recorded in control (8.17) while in local hybrid cotton number of bud abscission was significantly less in NAA $30 \mathrm{ppm}$ at 80 DAS (5.50). However, it was at par with MC 200 ppm at 60 DAS (7.00), MC $200 \mathrm{ppm}$ at 80 DAS (6.33), Urea $2 \%$ at 60 DAS (6.33). Significantly highest number of bud abscission was recorded in control (7.50) (Table 3.2).

Interaction effect of different plant growth regulators on Bt. cotton and local hybrid cotton for flower abscission at 90 DAS

The effect of different plant growth regulators on Bt. cotton and local hybrid cotton at 90 DAS was found to be significant. In $\mathrm{Bt}$. cotton the number of flower abscission differed significantly among the treatments. Number of flower abscission was significantly less when NAA $30 \mathrm{ppm}$ was applied at 80 DAS (7.83). However, it was at par with NAA $30 \mathrm{ppm}$ at $60 \mathrm{DAS}(8.83), \mathrm{GA}_{3}$ $50 \mathrm{ppm}$ at $60 \mathrm{DAS}(10.83), \mathrm{GA}_{3} 50 \mathrm{ppm}$ at 80 DAS (9.50), MC 200 ppm at 60 DAS (13.00), MC 200 ppm at 80 DAS (12.17), Urea $2 \%$ at 
60 DAS (11.17), Urea $2 \%$ at 80 DAS (11.17). Significantly highest number of flower abscission was recorded in control (13.83) while in local hybrid cotton number of flower abscission was significantly less when NAA $30 \mathrm{ppm}$ was applied at 60 DAS (9.17). However, it was at par with $\mathrm{GA}_{3} 50 \mathrm{ppm}$ at 60 DAS (10.67), MC $200 \mathrm{ppm}$ at 60 DAS (13.50), MC $200 \mathrm{ppm}$ at 80 DAS (12.33), Urea $2 \%$ at 60 DAS (11.33) and Urea $2 \%$ at 80 DAS (11.00). Significantly highest number of flower abscission was recorded in control (14.50) (Table 3.3).

Interaction effect of different plant growth regulators on Bt. cotton and local hybrid cotton for flowers opened at 90 DAS

The effect of different plant growth regulators on Bt. cotton and local hybrid cotton on number of flower opened at 90 DAS was found to be significant. The significant effect on flower opening was found due to plant growth regulators applied to Bt. cotton. Highest numbers of flowers were opened when NAA $30 \mathrm{ppm}$ applied at 80 DAS (26.17).Significantly less number of flower openings was recorded in MC $200 \mathrm{ppm}$ at 80 DAS (19.50) while in local hybrid cotton number of flower openings was significantly higher in NAA $30 \mathrm{ppm}$ at 60 DAS (24.17) However, it was at par with NAA30 ppm at 80 DAS (22.67) and $\mathrm{GA}_{3}(50 \mathrm{ppm}$ at 80 DAS (22.50). Significantly less number of flower openings was recorded in Control (17.33) (Table 3.4).

Interaction effect of different plant growth regulators on $\mathrm{Bt}$. cotton and local hybrid cotton relative growth rate (RGR) at 60-90 DAS

The effect of different plant growth regulators on Bt. cotton and local hybrid cotton on relative growth rate was found to be significant. In Bt. cotton the higher RGR was recorded when NAA $30 \mathrm{ppm}$ sprayed at 80 DAS (0.0486). However, it was at par with NAA $(30 \mathrm{ppm}$ at 60 DAS $)$ (0.0482). Significantly lower RGR was recorded in control (0.0430). In local hybrid cotton the higher RGR was recorded with NAA $30 \mathrm{ppm}$ at 80 DAS (0.0476). However, it was at par with NAA $30 \mathrm{ppm}$ at 60 DAS $(0.0475)$ and $\mathrm{GA}_{3} \quad 50 \mathrm{ppm}$ at 60 DAS $(0.0471)$. Significantly lower RGR was recorded in control (0.0417) (Table 3.5).

Interaction effect of different plant growth regulators on Bt. cotton and local hybrid cotton net assimilation rate (NAR) at 60-90 DAS

The effect of different plant growth regulators on Bt. cotton and local hybrid cotton on net assimilation rate was found to be significant. In Bt. cotton the higher NAR was recorded when NAA $30 \mathrm{ppm}$ was sprayed at 80 DAS (0.124). However, it was at par with NAA 30 $\mathrm{ppm}$ at $60 \mathrm{DAS}(0.122), \mathrm{GA}_{3} 50 \mathrm{ppm}$ at 60 DAS (0.122), $\mathrm{GA}_{3} 50 \mathrm{ppm}$ at 80 DAS (0.123), Urea $2 \%$ at 60 DAS (0.121) and Urea $2 \%$ at 80 DAS (0.122). Significantly lower NAR was recorded in control (0.112). In case of local hybrid cotton the higher NAR was recorded in NAA $30 \mathrm{ppm}$ at 80 DAS (0.120). However, it was at par with NAA 30 ppm at 60 DAS (0.118), $\mathrm{GA}_{3} 50 \mathrm{ppm}$ at 60 DAS (0.118), GA $\mathrm{GA}_{3} 50 \mathrm{ppm}$ at 80 DAS (0.119), MC $200 \mathrm{ppm}$ at 80 DAS (0.117) and Urea $2 \%$ at 80 DAS (0.118). Significantly lower NAR was recorded in control (0.104) (Table 3.6).

Interaction effect of different plant growth regulators on Bt. cotton and local hybrid cotton leaf area index (LAI) at 90 DAS

The effect of different plant growth regulators on Bt. cotton and local hybrid cotton on leaf area index was found to be significant. In Bt. cotton the higher leaf area index was recorded 
with NAA $30 \mathrm{ppm}$ at 80 DAS (1.85). Significantly lower leaf area index was recorded in MC 200 ppm at 60 DAS (1.18). In local hybrid cotton the higher leaf area index was recorded with MC $200 \mathrm{ppm}$ at 60 DAS (1.55). However, it was at par with NAA 30 ppm at 60 DAS (1.51), NAA $30 \mathrm{ppm}$ at 80 DAS (1.34), $\mathrm{GA}_{3} 50 \mathrm{ppm}$ at 60 DAS (1.47), $\mathrm{GA}_{3} 50 \mathrm{ppm}$ at 80 DAS (1.41), Urea $2 \%$ at 60 DAS (1.38), Urea $2 \%$ at 80 DAS (1.41) (Table 3.7).

Interaction effect of different plant growth regulators on Bt. cotton and local hybrid cotton chlorophyll content at 90 DAS

The effect of different plant growth regulators on Bt. cotton and local hybrid cotton on chlorophyll content was found to be significant. In Bt. cotton the higher chlorophyll content was recorded with $\mathrm{MC}$ 200 ppm applied at 80 DAS (1.56). Significantly lower chlorophyll content was recorded with control (1.30). In local hybrid cotton the higher chlorophyll content was recorded with MC 200 ppm at 80 DAS (1.44). However, it was at par with MC $200 \mathrm{ppm}$ at 60 DAS (1.42), $\mathrm{GA}_{3} 50 \mathrm{ppm}$ at 60 DAS (1.37), $\mathrm{GA}_{3} 50 \mathrm{ppm}$ at 80 DAS (1.38),
NAA30 ppm at 60 DAS (1.34), NAA30 ppm at 80 DAS (1.35), Urea $2 \%$ at 60 DAS (1.34) and Urea $2 \%$ at 80 DAS (1.35). Significantly lower chlorophyll content was recorded in control (1.09) (Table 3.8).

Interaction effect of different plant growth regulators on Bt. cotton and local hybrid cotton on seed cotton yield per plant (g plant $^{-1}$ )

The effect of different plant growth regulators on Bt. cotton and local hybrid cotton on seed cotton yield per plant was found to be significant. In Bt. cotton the higher seed cotton yield per plant was recorded with the spraying of NAA $30 \mathrm{ppm}$ at 80 DAS (70.03).

However, it was at par with NAA $30 \mathrm{ppm}$ at 60 DAS (66.50), $\mathrm{GA}_{3} 50 \mathrm{ppm}$ at $60 \mathrm{DAS}$ (67.65) and $\mathrm{GA}_{3} 50 \mathrm{ppm}$ at 80 DAS (68.05). Significantly lower seed cotton yield per plant was recorded in Control (51.70). In local hybrid cotton the higher seed cotton yield per plant was recorded with the application of NAA $30 \mathrm{ppm}$ at 80 DAS (61.00). However, it was at par with NAA $30 \mathrm{ppm}$ at 60 DAS (60.33) and $\mathrm{GA}_{3} 50 \mathrm{ppm}$ at 80 DAS (Table 3.9).

Table.1 Physico-chemical properties of soil of experimental field

\begin{tabular}{|c|c|c|c|c|c|}
\hline \multirow{2}{*}{$\begin{array}{l}\text { Sr. } \\
\text { No. }\end{array}$} & \multirow{2}{*}{\multicolumn{2}{|c|}{ Properties }} & \multicolumn{2}{|c|}{ Soil depth (cm) } & \multirow[t]{2}{*}{ Method employed } \\
\hline & & & $0-15$ & $15-30$ & \\
\hline [A] & \multicolumn{5}{|c|}{ PHYSICAL PROPERTIES } \\
\hline & (a) & Sand $(\%)$ & 84.90 & 84.98 & \multirow{4}{*}{$\begin{array}{l}\text { International Pipette Method } \\
\text { (Piper, 1966) }\end{array}$} \\
\hline & (b) & Silt $(\%)$ & 5.55 & 5.47 & \\
\hline & (c) & Clay $(\%)$ & 9.29 & 9.47 & \\
\hline & (d) & Soil texture & \multicolumn{2}{|c|}{ Loamy sand } & \\
\hline [B] & \multicolumn{5}{|c|}{ CHEMICAL PROPERTIES } \\
\hline & (a) & $\begin{array}{l}\text { Soil pH (1:2.5, Soil: } \\
\text { Water Ratio) }\end{array}$ & 7.6 & 7.4 & Potentiometric method (Jackson, 1978) \\
\hline & (b) & $\mathrm{EC}\left(\mathrm{dSm}^{-1}\right.$ at $\left.25^{\circ} \mathrm{C}\right)$ & 0.13 & 0.18 & Schofield method (Jackson, 1978) \\
\hline & (c) & Organic carbon $(\%)$ & 0.17 & 0.15 & $\begin{array}{l}\text { Weakley and Black's rapid titration method } \\
\text { (Jackson, 1978) }\end{array}$ \\
\hline & (d) & Available $\mathrm{N}\left(\mathrm{kg} \mathrm{ha}^{-1}\right)$ & 149 & 138 & Alkaline Permanganate method (Jackson, 1978) \\
\hline & (e) & Available $\mathrm{P}_{2} \mathrm{O}_{5}\left(\mathrm{~kg} \mathrm{ha}^{-1}\right)$ & 29.24 & 32.93 & Olsen's Method (Jackson, 1978) \\
\hline & (f) & Available $\mathrm{K}_{2} \mathrm{O}\left(\mathrm{kg} \mathrm{ha}^{-1}\right)$ & 287 & 279 & Flame photometer method (Jackson, 1978) \\
\hline
\end{tabular}


Table.2 Treatment combinations

\begin{tabular}{|c|c|c|c|}
\hline Treatments & $\begin{array}{l}\text { Treatment combinations } \\
\end{array}$ & Dose & Time of Spray \\
\hline $\mathrm{T}_{1}$ & NAA (1-naphthalene acetic acid) & $30 \mathrm{ppm}$ & $60 \mathrm{DAS}$ \\
\hline$\overline{T_{2}}$ & NAA (1-naphthalene acetic acid) & $30 \mathrm{ppm}$ & $80 \mathrm{DAS}$ \\
\hline $\mathrm{T}_{3}$ & $\mathrm{GA}_{3}$ (gibberellic acid) & $50 \mathrm{ppm}$ & 60 DAS \\
\hline $\mathrm{T}_{4}$ & $\mathrm{GA}_{3}$ (gibberellic acid) & $50 \mathrm{ppm}$ & 80 DAS \\
\hline $\mathrm{T}_{5}$ & $\begin{array}{l}\text { Mepiquat chloride (N, N-dimethyl piperdinium } \\
\text { chloride) }\end{array}$ & $200 \mathrm{ppm}$ & $60 \mathrm{DAS}$ \\
\hline $\mathrm{T}_{6}$ & $\begin{array}{l}\text { Mepiquat chloride (N, N-dimethyl piperdinium } \\
\text { chloride) }\end{array}$ & $200 \mathrm{ppm}$ & 80 DAS \\
\hline $\mathrm{T}_{7}$ & Urea & $2 \%$ & 60 DAS \\
\hline $\mathrm{T}_{8}$ & Urea & $2 \%$ & 80 DAS \\
\hline $\mathrm{T}_{9}$ & Control (No spray) & & \\
\hline $\mathrm{T}_{10}$ & NAA (1-naphthalene acetic acid) & $30 \mathrm{ppm}$ & 60 DAS \\
\hline$T_{11}$ & NAA (1-naphthalene acetic acid) & $30 \mathrm{ppm}$ & 80 DAS \\
\hline $\mathrm{T}_{12}$ & $\mathrm{GA}_{3}$ (gibberellic acid) & $50 \mathrm{ppm}$ & $60 \mathrm{DAS}$ \\
\hline$T_{13}$ & $\mathrm{GA}_{3}$ (gibberellic acid) & $50 \mathrm{ppm}$ & 80 DAS \\
\hline $\mathrm{T}_{14}$ & $\begin{array}{l}\text { Mepiquat chloride (N, N-dimethyl piperdinium } \\
\text { chloride) }\end{array}$ & $200 \mathrm{ppm}$ & 60 DAS \\
\hline $\mathrm{T}_{15}$ & $\begin{array}{l}\text { Mepiquat chloride (N, N-dimethyl piperdinium } \\
\text { chloride) }\end{array}$ & $200 \mathrm{ppm}$ & 80 DAS \\
\hline $\mathrm{T}_{16}$ & Urea & $2 \%$ & 60 DAS \\
\hline $\mathrm{T}_{17}$ & Urea & $2 \%$ & 80 DAS \\
\hline $\mathrm{T}_{18}$ & Control (No spray) & & \\
\hline
\end{tabular}

\begin{tabular}{|c|c|c|c|c|c|}
\hline \multicolumn{3}{|c|}{$\begin{array}{l}\text { Table.3.1 Interaction effect of plant growth } \\
\text { regulators on plant height }(\mathrm{cm}) \text { at } 90 \text { DAS }\end{array}$} & \multicolumn{3}{|c|}{$\begin{array}{l}\text { Table.3.2 Interaction effect of plant growth } \\
\text { regulators on number of bud abscission at } 9 \\
\text { DAS }\end{array}$} \\
\hline & \multicolumn{2}{|c|}{90 DAS } & & \multicolumn{2}{|c|}{$90 \mathrm{DAS}$} \\
\hline & $\mathrm{Bt}$ & Non Bt & & $\mathrm{Bt}$ & Non Bt \\
\hline $\mathrm{T}_{1}$ - NAA 30 ppm 60DAS & 92.00 & 92.08 & $\mathrm{~T}_{1}$ - NAA $30 \mathrm{ppm}$ 60DAS & 5.17 & 6.00 \\
\hline $\mathrm{T}_{2-}$-NAA $30 \mathrm{ppm} 80 \mathrm{DAS}$ & 95.33 & 91.92 & $\mathrm{~T}_{2-}$-NAA $30 \mathrm{ppm} 80 \mathrm{DAS}$ & 4.83 & 5.50 \\
\hline $\mathrm{T}_{3}-\mathrm{GA}_{3} 50 \mathrm{ppm} 60 \mathrm{DAS}$ & 89.67 & 92.00 & $\mathrm{~T}_{3^{-}} \mathrm{GA}_{3} 50 \mathrm{ppm} 60 \mathrm{DAS}$ & 5.50 & 6.17 \\
\hline $\mathrm{T}_{4^{-}}-\mathrm{GA}_{3} 50 \mathrm{ppm} 80 \mathrm{DAS}$ & 93.00 & 90.50 & $\mathrm{~T}_{4^{-}} \mathrm{GA}_{3} 50 \mathrm{ppm} 80 \mathrm{DAS}$ & 5.50 & 5.83 \\
\hline $\mathrm{T}_{5}-\mathrm{MC} 200 \mathrm{ppm} 60 \mathrm{DAS}$ & 84.33 & 81.00 & $\mathrm{~T}_{5}-\mathrm{MC} 200 \mathrm{ppm} 60 \mathrm{DAS}$ & 6.00 & 7.00 \\
\hline $\mathrm{T}_{6}-\mathrm{MC} 200 \mathrm{ppm} 80 \mathrm{DAS}$ & 85.25 & 82.73 & $\mathrm{~T}_{6}-\mathrm{MC} 200 \mathrm{ppm} 80 \mathrm{DAS}$ & 6.50 & 6.33 \\
\hline $\mathrm{T}_{7^{-}}$Urea $2 \% 60 \mathrm{DAS}$ & 85.67 & 84.00 & $\mathrm{~T}_{7}$ - Urea $2 \% 60 \mathrm{DAS}$ & 6.17 & 6.33 \\
\hline $\mathrm{T}_{8}$ - Urea $2 \% 80 \mathrm{DAS}$ & 86.00 & 88.00 & $\mathrm{~T}_{8}$ - Urea $2 \% 80 \mathrm{DAS}$ & 5.83 & 6.17 \\
\hline $\mathrm{T}_{9^{-}}$Control (No Spray) & 85.33 & 66.67 & $\mathrm{~T}_{9^{-}}$Control (No Spray) & 8.17 & 7.50 \\
\hline S.Em \pm & \multicolumn{2}{|c|}{2.822} & S.Em \pm & \multicolumn{2}{|c|}{0.244} \\
\hline C.D. at $5 \%$ & \multicolumn{2}{|c|}{8.11} & C.D. at $5 \%$ & \multicolumn{2}{|c|}{0.70} \\
\hline C. V\% & \multicolumn{2}{|c|}{5.62} & C. V\% & \multicolumn{2}{|c|}{6.89} \\
\hline
\end{tabular}




\begin{tabular}{|c|c|c|c|c|c|}
\hline \multicolumn{3}{|c|}{$\begin{array}{l}\text { Table.3.3 Interaction effect of plant growth } \\
\text { regulators on number of flowers abscission at } \\
90 \text { DAS. }\end{array}$} & \multicolumn{3}{|c|}{$\begin{array}{l}\text { Table.3.4 Interaction effect of plant growth } \\
\text { regulators on number of flowers opened at } 9 \\
\text { DAS. }\end{array}$} \\
\hline & \multirow{2}{*}{\multicolumn{2}{|c|}{90 DAS }} & & \multirow{2}{*}{\multicolumn{2}{|c|}{90 DAS }} \\
\hline & & & & & \\
\hline & $\mathrm{Bt}$ & Non Bt & & \multirow{2}{*}{\begin{tabular}{|c|}
$\mathrm{Bt}$ \\
23.50 \\
\end{tabular}} & Non Bt \\
\hline $\mathrm{T}_{1}$ - NAA $30 \mathrm{ppm}$ 60DAS & 8.83 & 9.17 & $\mathrm{~T}_{1}$ - NAA $30 \mathrm{ppm}$ 60DAS & & \multirow{2}{*}{$\begin{array}{l}24.17 \\
22.67\end{array}$} \\
\hline $\mathrm{T}_{2-}$-NAA $30 \mathrm{ppm} 80 \mathrm{DAS}$ & 7.83 & 10.00 & \multirow{2}{*}{ T$_{2-}$ NAA $30 \mathrm{ppm} 80 \mathrm{DAS}$} & 26.17 & \\
\hline $\mathrm{T}_{3}-\mathrm{GA}_{3} 50 \mathrm{ppm} 60 \mathrm{DAS}$ & 10.83 & 10.67 & & 23.33 & 20.67 \\
\hline $\mathrm{T}_{4}-\mathrm{GA}_{3} 50 \mathrm{ppm} 80 \mathrm{DAS}$ & 9.50 & 9.17 & $\mathrm{~T}_{3}-\mathrm{GA}_{3} 50 \mathrm{ppm} 60 \mathrm{DAS}$ & 23.17 & 22.50 \\
\hline $\mathrm{T}_{5^{-}}$MC $200 \mathrm{ppm} 60$ DAS & 13.00 & 13.50 & $\mathrm{~T}_{5^{-}}$MC $200 \mathrm{ppm} 60$ DAS & 20.83 & 19.00 \\
\hline $\mathrm{T}_{6}-\mathrm{MC} 200 \mathrm{ppm} 80 \mathrm{DAS}$ & 12.17 & 12.33 & \multirow{2}{*}{$\mathrm{T}_{6}-\mathrm{MC} 200 \mathrm{ppm} 80 \mathrm{DAS}$} & 19.50 & 20.83 \\
\hline $\mathrm{T}_{7}$ - Urea $2 \% 60 \mathrm{DAS}$ & 11.17 & $\begin{array}{l}11.33 \\
1100\end{array}$ & & 20.17 & 20.50 \\
\hline $\mathrm{T}_{8}$ - Urea $2 \% 80$ DAS & $\begin{array}{l}11.17 \\
1383\end{array}$ & $\begin{array}{l}11.00 \\
1450\end{array}$ & $\mathrm{~T}_{8}$ - Urea $2 \% 80 \mathrm{DAS}$ & 21.00 & 20.83 \\
\hline $\begin{array}{l}\mathrm{T}_{9^{-}} \text {Control (No Spray) } \\
\text { S.Em+ }\end{array}$ & \multicolumn{2}{|c|}{0.347} & $\mathrm{~T}_{9^{-}}$Control (No Spray) & 20.67 & 17.33 \\
\hline $\begin{array}{l}\text { S.Em } \pm \\
\text { C.D. at } 5 \%\end{array}$ & \multicolumn{2}{|c|}{1.00} & S.Em \pm & \multicolumn{2}{|c|}{0.78} \\
\hline C. V\% & \multirow{2}{*}{\multicolumn{2}{|c|}{5.40}} & C.D. at $5 \%$ & \multicolumn{2}{|c|}{2.24} \\
\hline & & & C. V\% & \multicolumn{2}{|c|}{6.29} \\
\hline
\end{tabular}

Table.3.5 Interaction effect of plant growth regulators on relative growth rate $\left(\mathrm{g} \mathrm{g}^{-1}\right.$ day $\left.^{-1}\right)$ at 90 DAS.

\begin{tabular}{|l|c|c|}
\hline \multirow{2}{*}{} & \multicolumn{2}{|c|}{$60-90 \mathrm{DAS}$} \\
\cline { 2 - 3 } & $\mathrm{Bt}$ & Non Bt \\
\hline $\mathrm{T}_{1^{-}}$NAA 30 ppm 60DAS & 0.0482 & 0.0475 \\
\hline $\mathrm{T}_{2-}$-NAA 30 ppm 80 DAS & 0.0486 & 0.0476 \\
\hline $\mathrm{T}_{3^{-}} \mathrm{GA}_{3}$ 50 ppm 60 DAS & 0.0471 & 0.0471 \\
\hline $\mathrm{T}_{4^{-}} \mathrm{GA}_{3}$ 50 ppm 80 DAS & 0.0473 & 0.0464 \\
\hline $\mathrm{T}_{5^{-}}$MC 200 ppm 60 DAS & 0.0433 & 0.0431 \\
\hline $\mathrm{T}_{6}$ - MC 200 ppm 80 DAS & 0.0435 & 0.0432 \\
\hline $\mathrm{T}_{7^{-}}$Urea 2 \% 60 DAS & 0.0450 & 0.0445 \\
\hline $\mathrm{T}_{8^{-}}$Urea 2 \% 80 DAS & 0.0453 & 0.0451 \\
\hline $\mathrm{T}_{9^{-}}$Control (No Spray) & 0.0430 & 0.0417 \\
\hline S.Em \pm & \multicolumn{2}{|c|}{0.0002} \\
\hline C.D. at 5\% & \multicolumn{2}{|c|}{0.0006} \\
\hline C. V \% & \multicolumn{2}{|c|}{} \\
\hline
\end{tabular}

Table.3.6 Interaction effect of plant growth regulators on net assimilation rate $\left(\mathrm{g}^{-1} \mathrm{dm}^{-2}\right.$ day $^{-1}$ ) at 90 DAS.

\begin{tabular}{|c|c|c|}
\hline & \multicolumn{2}{|c|}{$60-90$ DAS } \\
\hline & $\mathrm{Bt}$ & Non Bt \\
\hline $\mathrm{T}_{1}$ - NAA $30 \mathrm{ppm}$ 60DAS & 0.122 & 0.118 \\
\hline 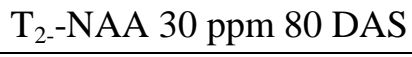 & 0.124 & 0.120 \\
\hline $\mathrm{T}_{3}-\mathrm{GA}_{3} 50 \mathrm{ppm} 60 \mathrm{DAS}$ & 0.122 & 0.118 \\
\hline $\mathrm{T}_{4^{-}} \mathrm{GA}_{3} 50 \mathrm{ppm} 80 \mathrm{DAS}$ & 0.123 & 0.119 \\
\hline $\mathrm{T}_{5^{-}} \mathrm{MC} 200 \mathrm{ppm} 60 \mathrm{DAS}$ & 0.118 & 0.115 \\
\hline $\mathrm{T}_{6^{-}} \mathrm{MC} 200 \mathrm{ppm} 80 \mathrm{DAS}$ & 0.119 & 0.117 \\
\hline $\mathrm{T}_{7}$ - Urea $2 \% 60 \mathrm{DAS}$ & 0.121 & 0.115 \\
\hline $\mathrm{T}_{8}$ - Urea $2 \% 80 \mathrm{DAS}$ & 0.122 & 0.118 \\
\hline $\mathrm{T}_{9^{-}}$Control (No Spray) & 0.112 & 0.104 \\
\hline S.Em \pm & \multicolumn{2}{|c|}{0.0009} \\
\hline C.D. at $5 \%$ & \multicolumn{2}{|c|}{0.003} \\
\hline C. V\% & \multicolumn{2}{|c|}{1.30} \\
\hline
\end{tabular}


Table.3.7 Interaction effect of plant growth regulators on leaf area index (LAI) at 90 DAS.

\begin{tabular}{|c|c|c|}
\hline & \multicolumn{2}{|c|}{90 DAS } \\
\hline & $\mathrm{Bt}$ & Non Bt \\
\hline $\mathrm{T}_{1}$ - NAA 30 ppm 60 DAS & 1.57 & 1.51 \\
\hline $\mathrm{T}_{2-}$-NAA $30 \mathrm{ppm} 80 \mathrm{DAS}$ & 1.85 & 1.34 \\
\hline $\mathrm{T}_{3}-\mathrm{GA}_{3} 50 \mathrm{ppm} 60 \mathrm{DAS}$ & 1.44 & 1.47 \\
\hline $\mathrm{T}_{4}-\mathrm{GA}_{3} 50 \mathrm{ppm} 80 \mathrm{DAS}$ & 1.61 & 1.41 \\
\hline $\mathrm{T}_{5^{-}} \mathrm{MC} 200 \mathrm{ppm} 60 \mathrm{DAS}$ & 1.18 & 1.55 \\
\hline $\mathrm{T}_{6}-\mathrm{MC} 200 \mathrm{ppm} 80 \mathrm{DAS}$ & 1.60 & 1.14 \\
\hline $\mathrm{T}_{7^{-}}$Urea $2 \% 60$ DAS & 1.39 & 1.38 \\
\hline $\mathrm{T}_{8}$ - Urea $2 \% 80 \mathrm{DAS}$ & 1.42 & 1.41 \\
\hline $\mathrm{T}_{9^{-}}$Control (No Spray) & 1.33 & 1.38 \\
\hline S.Em \pm & \multicolumn{2}{|c|}{0.076} \\
\hline C.D. at $5 \%$ & \multicolumn{2}{|c|}{0.22} \\
\hline C. V\% & \multicolumn{2}{|c|}{9.13} \\
\hline
\end{tabular}

Table.3.8 Interaction effect of plant growth regulators on chlorophyll content $\left(\mathrm{mg} \mathrm{g}_{-}{ }^{1}\right.$ fresh weight) at 90 DAS.

\begin{tabular}{|c|c|c|}
\hline & \multicolumn{2}{|c|}{$90 \mathrm{DAS}$} \\
\hline & $\mathrm{Bt}$ & Non Bt \\
\hline $\mathrm{T}_{1}$ - NAA $30 \mathrm{ppm}$ 60DAS & 1.31 & 1.34 \\
\hline $\mathrm{T}_{2-\mathrm{NAA}} 30 \mathrm{ppm} 80 \mathrm{DAS}$ & 1.33 & 1.35 \\
\hline $\mathrm{T}_{3}-\mathrm{GA}_{3} 50 \mathrm{ppm} 60 \mathrm{DAS}$ & 1.37 & 1.37 \\
\hline $\mathrm{T}_{4}-\mathrm{GA}_{3} 50 \mathrm{ppm} 80 \mathrm{DAS}$ & 1.38 & 1.38 \\
\hline $\mathrm{T}_{5}-\mathrm{MC} 200 \mathrm{ppm} 60$ DAS & 1.42 & 1.42 \\
\hline $\mathrm{T}_{6}-\mathrm{MC} 200 \mathrm{ppm} 80 \mathrm{DAS}$ & 1.56 & 1.44 \\
\hline $\mathrm{T}_{7}$ - Urea $2 \% 60$ DAS & 1.36 & 1.34 \\
\hline $\mathrm{T}_{8}$ - Urea $2 \% 80 \mathrm{DAS}$ & 1.37 & 1.35 \\
\hline $\mathrm{T}_{9^{-}}$Control (No Spray) & 1.30 & 1.09 \\
\hline S.Em \pm & \multicolumn{2}{|c|}{0.036} \\
\hline C.D. at $5 \%$ & \multicolumn{2}{|c|}{0.104} \\
\hline C. V\% & \multicolumn{2}{|c|}{4.63} \\
\hline
\end{tabular}

Table.3.9 Interaction effect of plant growth regulators on seed cotton yield (gm. / ha) of cotton.
Table.4 Interaction effect of plant growth regulators on biological yield (gm.) of cotton.

\begin{tabular}{|l|c|c|}
\hline \multirow{2}{*}{} & \multicolumn{2}{|c|}{$\begin{array}{c}\text { Seed cotton yield } \\
\text { (gm. / ha })\end{array}$} \\
\cline { 2 - 3 } & $\mathrm{Bt}$ & Non Bt \\
\hline $\mathrm{T}_{1}$ - NAA 30 ppm 60DAS & 66.50 & 60.33 \\
\hline $\mathrm{T}_{2}-\mathrm{NAA} 30 \mathrm{ppm}$ 80 DAS & 70.03 & 61.00 \\
\hline $\mathrm{T}_{3}-\mathrm{GA}_{3} 50 \mathrm{ppm}$ 60 DAS & 67.65 & 52.25 \\
\hline $\mathrm{T}_{4}-\mathrm{GA}_{3} 50 \mathrm{ppm}$ 80 DAS & 68.05 & 56.02 \\
\hline $\mathrm{T}_{5}$ - MC 200 ppm 60 DAS & 47.28 & 49.15 \\
\hline $\mathrm{T}_{6}$ - MC 200 ppm 80 DAS & 57.03 & 42.00 \\
\hline $\mathrm{T}_{7}$ - Urea 2 \% 60 DAS & 53.35 & 47.48 \\
\hline $\mathrm{T}_{8}$ - Urea 2 \% 80 DAS & 53.94 & 47.62 \\
\hline $\mathrm{T}_{9}$ - Control (No Spray) & 51.70 & 42.13 \\
\hline S.Em \pm & \multicolumn{2}{|c|}{2.05} \\
\hline C.D. at 5\% & \multicolumn{2}{|c|}{5.89} \\
\hline C. V \% & 6.43 \\
\hline
\end{tabular}

\begin{tabular}{|c|c|c|}
\hline & \multicolumn{2}{|c|}{$\begin{array}{l}\text { Biological yield } \\
\text { (gm.) }\end{array}$} \\
\hline & $\mathrm{Bt}$ & Non Bt \\
\hline $\mathrm{T}_{1}$ - NAA $30 \mathrm{ppm}$ 60DAS & 241.49 & 236.37 \\
\hline $\mathrm{T}_{2-}$-NAA $30 \mathrm{ppm} 80 \mathrm{DAS}$ & 249.96 & 233.35 \\
\hline $\mathrm{T}_{3}-\mathrm{GA}_{3} 50 \mathrm{ppm} 60 \mathrm{DAS}$ & 249.37 & 209.53 \\
\hline $\mathrm{T}_{4^{-}} \mathrm{GA}_{3} 50 \mathrm{ppm} 80 \mathrm{DAS}$ & 245.96 & 213.00 \\
\hline $\mathrm{T}_{5^{-}}$MC $200 \mathrm{ppm} 60$ DAS & 211.63 & 221.22 \\
\hline $\mathrm{T}_{6^{-}} \mathrm{MC} 200 \mathrm{ppm} 80 \mathrm{DAS}$ & 234.86 & 186.87 \\
\hline $\mathrm{T}_{7^{-}}$Urea $2 \% 60$ DAS & 217.90 & 199.00 \\
\hline $\mathrm{T}_{8}$ - Urea $2 \% 80$ DAS & 223.91 & 206.02 \\
\hline $\mathrm{T}_{9^{-}}$Control (No Spray) & 224.34 & 201.91 \\
\hline S.Em \pm & \multicolumn{2}{|c|}{2.12} \\
\hline C.D. at $5 \%$ & \multicolumn{2}{|c|}{6.11} \\
\hline C. V\% & \multicolumn{2}{|c|}{1.65} \\
\hline
\end{tabular}


Table.4.1 Interaction effect of plant growth regulators on harvest index (\%) of cotton.

\begin{tabular}{|l|c|c|}
\hline \multirow{2}{*}{} & \multicolumn{2}{|c|}{ Harvest index (\%) } \\
\cline { 2 - 3 } & $\mathrm{Bt}$ & Non Bt \\
\hline $\mathrm{T}_{1}$ - NAA 30 ppm 60DAS & 37.74 & 34.28 \\
\hline $\mathrm{T}_{2}-\mathrm{NAA} 30 \mathrm{ppm}$ 80 DAS & 38.86 & 35.34 \\
\hline $\mathrm{T}_{3}$ - $\mathrm{GA}_{3}$ 50 ppm 60 DAS & 37.08 & 33.99 \\
\hline $\mathrm{T}_{4}-\mathrm{GA}_{3}$ 50 ppm 80 DAS & 38.13 & 35.42 \\
\hline $\mathrm{T}_{5}$ - MC 200 ppm 60 DAS & 28.00 & 28.79 \\
\hline $\mathrm{T}_{6}$ - MC 200 ppm 80 DAS & 32.26 & 29.28 \\
\hline $\mathrm{T}_{7}$ - Urea 2 \% 60 DAS & 32.38 & 31.62 \\
\hline $\mathrm{T}_{8}$ - Urea 2 \% 80 DAS & 31.69 & 31.17 \\
\hline $\mathrm{T}_{9}$ - Control (No Spray) & 29.83 & 27.22 \\
\hline S.Em \pm & \multicolumn{2}{|c|}{0.48} \\
\hline C.D. at 5\% & \multicolumn{2}{|c|}{1.38} \\
\hline C. V \% & \multicolumn{2}{|c|}{2.53} \\
\hline
\end{tabular}

NAA - Naphthalene acetic acid MC - Mepiquat chloride $\mathrm{GA}_{3}$ - Gibberellic acid DAS - Days after sowing

Interaction effect of different plant growth regulators on Bt. cotton and local hybrid cotton on biological yield $(\mathrm{g})$

The effect of different plant growth regulators on Bt. cotton and local hybrid cotton on biological yield was found to be significant. In Bt. cotton the higher biological yield was recorded with NAA $30 \mathrm{ppm}$ at 80 DAS (249.96) and it was at par with $\mathrm{GA}_{3} 50 \mathrm{ppm}$ at 60 DAS (249.37) and $\mathrm{GA}_{3} 50 \mathrm{ppm}$ at $80 \mathrm{DAS}$ (245.96). In local hybrid cotton the higher biological yield was recorded with NAA 30 ppm at 60 DAS (236.37). However, it was at par with NAA $30 \mathrm{ppm}$ at 80 DAS (233.35). Significantly lower biological yield was recorded in MC $200 \mathrm{ppm}$ at 80 DAS (186.87) (Table 4).

Interaction effect of different plant growth regulators on Bt. cotton and local hybrid cotton on harvest index

The effect of different plant growth regulators on Bt. cotton and local hybrid cotton on harvest index was found to be significant. In Bt. cotton the high harvest index was recorded in NAA $30 \mathrm{ppm}$ at 80 DAS (38.86) and it was at par with $\mathrm{GA}_{3} 50 \mathrm{ppm}$ at $80 \mathrm{DAS}$ (38.13). In local hybrid cotton the high harvest index was recorded in $\mathrm{GA}_{3} 50 \mathrm{ppm}$ at 80 DAS (35.42). However, it was at par with NAA $30 \mathrm{ppm}$ at 60 DAS (34.28) and NAA 30 ppm at 80 DAS (35.34). Significantly low harvest index was recorded in control (27.22) (Table 4.1).

The increase in plant height of cotton plants sprayed with NAA could mainly be attributed to its physiological role in stimulation of cell elongation and promotion of cell division which results into stem elongation. This is in agreement with Patel (1993) who reported that the application of NAA @ 20 ppm increased plant height in cotton. Such increase in plant height due to NAA spray was also reported by Dastur and Prakash (1954) and Annapan and Aaron (1969), in cotton.

Application of MC @ 200 ppm decreased the plant height as compared to other treatments and this is similar to the results of Walter $e t$ al., (1980), Sawan and Sakr (1990) and Reddy et al., (1996). This mechanism of reduction in the cell elongation is because of inhibitory effect of mepiquat chloride in the 
biosynthetic pathway of gibberellins in the plant body (Reddy et al., 1996). Similarly, Wilhelm Rademacher (2000) reported that onium compounds, such as chloromequat chloride, MC and AMO-1618, which block the cyclassescopalyl diphosphate synthase and ent-kaurene synthase involved in the early steps of GA biosynthesis.

The growth promoter NAA @ 30 ppm application recorded significantly higher leaf area index. These results are similar to that of Eid and Al-Abdel (1985) and Dhillon et al., (1992). However, MC treatments significantly reduced the leaf area index as compared to NAA concentrations. This variation in leaf area index could be attributed to differential mode of growth promoters and retardants (Walter et al., 1980, York., 1983, Stewart 2005, Hake et al., 1991 and Mangal Prasad and Rajendra Prasad, 1994). Reduction in LAI by growth retardants might also be due to increased juvenility. It also resulted in thicker mesophyll tissues compared to control which is associated with higher chlorophyll content thus making the leaves to be dark green in colour and photosynthetically active for longer period (Bhattand Nathan, 1970).

Foliar application of NAA @ 30 ppm significantly gave early and number of flowers opening compared to other treatments. The application of NAA increased the flowering percentage, reduced the abscission and increased the flower retention percentage, which in turn helped in getting higher yield of seed cotton.

The reduction in the abscission of intact buds and flowers per plant was observed. NAA completely counteracted the abscission promotive effect of ABA and thus reduced the shedding over the control. It was suggested that endogenous auxin content maybe playing a key role in the phenomenon of abscission and that a certain concentration might regulate the process (Varma, 1978).Growth parameters like RGR and NAR have been extensively used in recent years for better understanding of physiological basis of yield variation in crop plants. Increase in yield is not associated with increase in photosynthetic rate alone and it is difficult to find out clear cut answer for improving the yield potential.

The RGR was more during early stages and gradually decreased thereafter. This indicates that RGR in cotton is more closely associated with vegetative growth than seed cotton yield (Coy, 1976). At initial stage (60-90 DAS), higher RGR was recorded with NAA treatments. The increase in RGR by the application of growth regulators could be attributed to increased photosynthetic efficiency as a result of increased leaf thickness, higher chlorophyll content and efficient translocation of photosynthates (Joseph and Johnson, 2006).

In the present study, mepiquat chloride @ 200 ppm recorded the maximum total chlorophyll content. This is in agreement with the results of Bhatt and Ramanujam (1971) and Reddy et al., (1996). Bhatt and Nathan (1970) inferred that the application of growth retardants produced thicker leaf blades. This is in line with the results of More et al., (1993). In the present investigation, higher yields were obtained with NAA @ 30 ppm application.

This increased yield was due to higher seed cotton yield per plant. Several authors have also reported increased seed cotton yield due to NAA spray (Dastur and Bhatt, 1956; Bharadwaj andSanthanam, 1962; Sankaran and Balasubramanian, 1975; Jaganathan and Ireetharaj, 1982; Patel, 1993; Sawan et al., 1998). This was because of higher number of harvested bolls per plant and higher mean boll weight (Bharadwaj and Sharma, 1971 and Bhale et al., 1987). Biological yield is measured in terms of percent and is being 
utilized for the production of economic yield. Among the treatments, NAA (30 ppm) recorded the maximum biological yield.

Harvest index indicates the translocation efficiency of plants and is measured in terms of percent of dry matter being utilized for the production of economic yield. Among the treatments, NAA @ 30 ppm recorded the maximum harvest index. Harvest index was having significant positive correlation with yield. Basu and Bhatt (1987) reported that genetic improvement of harvest index would improve the seed cotton yield.

\section{References}

Annappan, R. S. and Aaron, D. S. (1969). Effect of alfa-napthalene acetic acid on Karuganni Cotton. Madras Agricultural Journal, 56: 488-491.

Basu, A. K. and Bhatt, M. G. (1987).Variability of harvest index in upland cotton. Indian Journal of Agricultural Sciences, 57: 219-288.

Bhale, N. L., Rao, M. R. K. and Deshmukh, R, K. (1987).New approach to reduce the cost of hybrid seed production. I. Use of hormones. Current Science, 56: 776-778.

Bharadwaj, S. N. and Santhanam, V. (1962). Physiological studies on bud and boll shedding in cotton. Indian Cotton Growing review, 16: 203-212.

Bhatt, J. G. and Nathan, A. R. S. (1970). Changes in foliar anatomy of cotton caused by growth retardants. Indian Journal of Agricultural Sciences, 40: 1142-1196.

Bhatt, J. G. and Ramanujam, T. (1971). Some responses of a short branched cotton varieties to gibberellin. Cotton Growing Review, 48: 136-139.

Das, V. L. D. (1982). Studies on Egyptian cotton ( $G$. barbadense L.) and their yield components. Cotton Development,
11: 7-24

Dastur, R. H. and Bhatt, J. G.(1956).Effect of chemical hormones on the carbohydrate sand nitrogen contents of cotton plant. Indian Journal of Agricultural Sciences, 26:39-79.

Dastur, R. H. and Prakash, V.(1954). Response of the cotton plants to some growth regulating substances. Indian Cotton Growing Review, 8: 173-188.

Dastur, R. H. (1959). Physiological Studies on Cotton crop and their practical applications. Published by Indian Central Cotton Committee, Bombay, pp. 42-62.

Dhillon, G. S., Kler, D. S. and Raj, D. (1992). Effect of cycocel on the growth, abscissionand yield of cotton ( $G$. hirsutum L.). Journal of Australian Agricultural Sciences, 112: 200-201.

Eid, E. T. and Al-abdel, M. H. (1985). Effect of NAA on growth, yield components and chemical constituents of cotton plant and seeds of three Egyptian cotton varieties. Annual Agricultural Science, 30: 1031-1046.

Hake, K., T. Kerby, W. McCarty, D. O'neal, and Supak J. (1991). Physiology of Pix. Physiology Today Volume 2, Number 6.

Jackson, M. L. (1978). Soil Chemical Analysis, Prentice Hall of India Pvt. Ltd. New Delhi, pp.183-192.

Kiran Kumar, K. A. (2001). Effect of plant growth regulators on morphophysiological traits and yield attributes in hybrid cotton (Gossypium hirsutum L.). M. Sc. (Agri.) Thesis, University of Agricultural Sciences, Dharwad.

Mangal Prasad and Rajendra Prasad, (1994). Effect of some plant growth regulators in cotton. Indian Journal of Plant Physiology, 37: 109-110.

Panse, V. G. and Sukhatme, P. V.(1967). Statistical Methods for Agricultural Workers, ICAR Publication, New 
Delhi, pp. 167-174.

Patel, J. K. (1993). Response of cotton ( $G$. hirsutum) to triacontanol and naphthalene aceticacid sprays. Indian Journal of Agronomy, 38(1): 97-101.

Piper, C. S. (1966). Soil and Plant Analysis. Academic Press, New York, p. 368.

Reddy, A. R., Reddy, K. R. and Hodges, H. F. (1996). Mepiquat chloride (PIX) induced changes in photosynthesis and growth of cotton. Plant Growth Regulation, 20:179-189.

Sawan, Z. M., Sakr, R. A. and Ahmed, F. A.(1989). Effect of NAA on seed protein, oil and fatty acids of Egyptian cotton. Journal of American Oil Chemists Society, 66:1472-1474.

Sawan, Z. M., Sakr, R. A. and Momtaz, O. A. (1998). Effect of naphthalene acetic acid concentrations and the number of application on the yield components, yield and fibre properties of Egyptian cotton ( $G$. barbadense L.).Australian Journal of Agricultural Research, 49: 955-960.

Stewart, S. (2005). Suggested Guidelines for Plant Growth Regulator Use on Louisiana Cotton. Louisiana Cooperative Extension Service,
Publication Number 2919.

Varma, S. K. (1976). Effect of localized application of ascorbic acid and other plant regulators singly and in combination with ABA on boll shedding in cotton ( $G$. hirsutum L.). Indian Journal of Experimental Biology, 14: 305-308.

Varma, S. K. (1978). Interaction between kinetin and ABA in controlling abscission of flower buds and bolls of cotton ( $G$. hirsutum L.). Acta Botany Indica, 6: 52-57.

Walter, H., Gausman, H. W., Ritting, F. R., Namken, L. N., Escobar, D. E. and Rodriguez. (1980). Effect of mepiquat chloride on cotton plant leaf and canopy structure and dry weights of its components. Beltwide Cotton Production Research Conference Proceedings, USA, Nation Cotton Council of America, PP.32-35.

Wilhelm Rademacher. (2000). Growth retardants effects on gibberellin biosynthesis and other metabolic pathways. Annual Review of Plant Physiology and Plant Molecular Biology, 51: 501-531.

\section{How to cite this article:}

Sabale, S.S., G.R. Lahane and Dhakulkar, S.J. 2017. Effect of Various Plant Growth Regulators on Growth and Yield of Cotton (Gossypium hirsutum). Int.J.Curr.Microbiol.App.Sci. 6(11): 978-989. doi: https://doi.org/10.20546/ijcmas.2017.611.115 